\title{
Semantic Fusion for Query Processing in Grid Environment
}

\author{
Jinguang $\mathrm{Gu}$ \\ ${ }^{1}$ College of Computer Science and Engineering, \\ Southeast University, Nanjing 210096, China \\ sam@seu.edu.cn \\ ${ }^{2}$ College of Computer Science and Technology, \\ Wuhan University of Science and Technology, Wuhan 430081, China
}

\begin{abstract}
To enable accessing web information at semantic level, this paper develops a semantic fusion mechanism in Mediator-Wrapper based grid environment to support ontology-based query planning with GAV style querying request. It employs semantic communication language to build mediator site for different virtual organizations (VO), and creates global semantic information for VOs. The procedure of ontology based semantic fusion is discussed in detail.
\end{abstract}

\section{Introduction}

We witness a rapid increase in the number of web information sources that are available online. The World-Wide Web(WWW), in particular , is a popular medium for interacting with such sources[1]. How to integrate and query distributed and heterogeneity information, especially semi-structured and nonstructured information is the problem we need to solve. Data grid technology is the standard means of realizing the needs. However, the studies in data grid technology still have the shortcomings as follows: 1)The flexibility of the grid technology is limited. Taking OGSA-DAI 2] for example, it only supports the limited related database and native XML database. However, most information on Internet comes from web-based semi-structured data environment, such as company web application and XML-based e-commerce platform; furthermore, OGSA-DAI does not have the effective mechanism for other data sources to be integrated into the grid environment. 2) The individual node in the grid environment may exist in varied semantic environment; different data resource is constructed in accordance with different semantic standard. The present data grid does not take into consideration the semantic heterogeneity among different nodes.

This paper proposes a semantic data grid (SDG for short) service to solve these two problems and discusses the semantic fusion mechanism in detail. SDG employs a mediator-wrapper framework to support different information sources and enable semantic information operation on different grid nodes 3 , and it uses semantic communication language to create mediator-wrapper structure 
dynamically for different virtual organizations(VO for short)on the grid, creates semantic fusion list on the mediator site to support Global-as-View(GAV) style querying request. This grid architecture uses a semantic grid adapter service to support semantic operation on the gird. The function of the wrapper of local grid nodes is to describe its semantics and its mapping relationship with other nodes, the information source of these nodes include both free and commercial databases, flat files services, web services or web based applications, HTML files and XML files, and the semantic information of every local gird node is described with the language based on its ontology. The mediator node constructs the global semantics of the local nodes, the semantic communication mechanism between the mediator and wrapper nodes is discussed in the following section. The remainder of this paper is structured as follows. Section 2 discusses the knowledge communication mechanism to support semantic querying and knowledge fusion. Section 3 discusses the procedure of ontology based semantic fusion. Section 4 summarizes the whole paper.

\section{Communication Mechanism with Semantic Grid}

It is very important to develop a knowledge communication and coordinating mechanism to support the ontology fusion and semantic query on different data grid nodes. This paper employs a Knowledge Communication and Manipulation Language for Semantic Grid, or KCML for short to support this mechanism, which is an extension of the KGOL 4 language. One function of KCML is to coordinate with each grid node to build the mediator-wrapper architecture dynamically. The other function is to build global knowledge on the mediator and enable semantic query. The communication language is build on SOAP, following the expression of SOAP's class XML, supporting SOAP over HTTP, HTTPS or other rock-bottom communication protocol. The language could describe as:

$K C M L::=\operatorname{Ver} \mid$ Operation $\mid$ Sender $\mid$ Receiver $\mid$ Language $\mid$ Content.

The field $V e r$ is for keeping Expanding, showing which version language was used. The new version language has compatibility downwards, supporting the old communication mechanism; Operation gives basic communication atom which will be described next; Content describes what is communicated; Sender defines sender's information, including user, address (such as IP ,e-mail,URL, port); $R e$ ceiver defines receiver's information (usually, receiver should be Web Service or Grid Service), including type (HOST, Web Service or Semantic Web Service), address(such as IP address, e-mail, URL, port, if receiver is Web Service, also including service address), identifier; language defines which language is used this communication, including RDF/RDFs, DAML+OIL, OWL etc. To illustrate communication atoms, we first define the ontology based knowledge on the mediators and wrappers.

Definition 1. A knowledge schema is a structure $K B:=\left(C_{K B}, R_{K B}, I, \iota_{C}, \iota_{R}\right)$ consisting of (1) two sets $C_{K B}$ and $R_{K B}$, (2) a set $I$ whose elements are called instance identifiers or instances, (3) a function $\iota_{C}: C_{K B} \rightarrow \Re(I)$ called concept instantiation, (4) a function $\iota_{R}: R_{K B} \rightarrow \Re\left(I^{+}\right)$called relation instantiation. 
The atom includes basic communication atoms and semantic fusion atoms. Basic communication atoms such as selection, join, union, minus and projection are discussed in paper [5] 6]. Semantic fusion atoms represent semantic matching and fusion procedure.

The mediator node constructs the global semantics of the local nodes based on ontology via ontology fusion mechanism[7] based on the ontology mapping patterns in gird environment, the patterns of ontology mapping can be categorized into four expressions: direct mapping, subsumption mapping, composition mapping and decomposition mapping [8], a mapping can be defined as:

Definition 2. A Ontology mapping is a structure $\mathcal{M}=(\mathcal{S}, \mathcal{D}, \mathcal{R}, v)$, where $\mathcal{S}$ denotes the concepts of source ontology, $\mathcal{D}$ denotes the concepts of target ontology, $\mathcal{R}$ denotes the relation of the mapping and $v$ denotes the confidence value of the mapping, $0 \leq v \leq 1$.

A direct mapping relates ontology concepts in distributed environment directly, and the cardinality of direct mapping could be one-to-one. A subsumption mapping is a 6-tuple $\mathcal{S}_{\mathcal{M}}=\left(\mathcal{D}_{m}, \mathcal{R}_{m}, \mathcal{B}_{m}, \preceq_{m}, \mathcal{I}_{m}, v\right)$, where $\mathcal{D}_{m}$ is a direct mapping expression; $\mathcal{R}_{m}$ is the first target concept, which is the most specialized ontology concept. The mapping between the source ontology and $\mathcal{R}_{m}$ is denoted as Root ontology concept mapping; $\mathcal{B}_{m}$ is the last target concept, which is the most generalized ontology concept. The mapping between the source ontology and $\mathcal{B}_{m}$ is denoted as Bottom ontology concept mapping; $\preceq_{m}$ is inclusion relation between target ontology concepts; $\mathcal{I}_{m}$ is the inverse mapping. Subsumption mapping is used to denote concept inclusion relation especially in the multiple IS-A inclusion hierarchy. The composition mapping is a 4tuple $\mathcal{C}_{\mathcal{M}}=\left(\mathcal{F}_{m}, \mathcal{A}_{m}, \mathcal{B}_{m}, v\right)$, where $\mathcal{F}_{m}$ is a direct mapping expression; $\mathcal{A}_{m}$ is chaining of role(s) between target ontology concepts; $\mathcal{B}_{m}$ is the last target symbol, which is the node of chaining target role(s), and composition mapping is used to map one concept to combined concepts. For example, the mapping address= contact (country, state, city, street, postcode $)$ is a composition mapping, in which the concept address is mapped to combined concept "contact, country, state, street, and postcode" of local schema elements. The decomposition mapping is a 4-tuple $\mathcal{C}_{\mathcal{M}}=\left(\mathcal{A}_{m}, \mathcal{B}_{m}, \mathcal{L}_{m}, v\right)$, where $\mathcal{A}_{m}$ is chaining of role(s) between source ontology concepts; $\mathcal{B}_{m}$ is the last target symbol, which is the node of chaining source role(s); $\mathcal{L}_{m}$ is a direct mapping expression. Decomposition mapping is used to map a combined concept to one local concept, and the example for the decomposition mapping is the reverse of the composition.

The KCML language must support the mapping patterns between different semantic nodes on gird, we use Match atom to support it, it can be defined as $M(c, d, r)=\left\{(x, y) \mid x \in \iota_{C}(c) \wedge y \in \iota_{C}(d) \wedge(x, y) \in \iota_{R}(r)\right\}$, where $c$ is different concept from $d, r$ is relationship of mapping.

The knowledge stored at mediator can be described as the ontology fusion connections list, which can be described as definition 3. The corresponding fusion connection lists of the mapping patterns can be denote as $\mathcal{F}_{c d}, \mathcal{F}_{c s}$ and $\mathcal{F}_{c c}$ respectively. 
Definition 3. Fusion Connection is a structure $\mathcal{F}_{c}\left(O_{1}: C_{1}, O_{2}: C_{2}, \ldots, O_{n}\right.$ : $\left.C_{n}, \mathcal{M}\right)$, where $C_{1}$ denotes a concept or concept set of ontology $O_{1}, C_{2}$ denotes a concept or concept set of Ontology $\mathrm{O}_{2}, \mathcal{M}$ denotes the mapping patterns between $C_{1}, C_{2}, \ldots$ and $C_{n}$.

\section{The Procedure of Semantic Fusion}

The function of Ontology fusion is to add connection tag between the concepts that have direct mapping relationship. In direct mapping $\mathcal{M}=(\mathcal{S}, \mathcal{D}, \mathcal{R}, v)$, the fusion connection adds connection tag between the elements of $\mathcal{S}$ and $\mathcal{D}$; in subsumption mapping $\mathcal{S}_{\mathcal{M}}=\left(\mathcal{D}_{m}, \mathcal{R}_{m}, \mathcal{B}_{m}, \preceq_{m}, \mathcal{I}_{m}, v\right)$, the fusion connection adds connection tag between the concepts which have mapping relation $\mathcal{D}_{m}$, and in composition mapping $\mathcal{C}_{\mathcal{M}}=\left(\mathcal{F}_{m}, \mathcal{A}_{m}, \mathcal{B}_{m}, v\right)$ or decomposition mapping $\mathcal{C}_{\mathcal{M}}=\left(\mathcal{A}_{m}, \mathcal{B}_{m}, \mathcal{L}_{m}, v\right)$, the fusion connection adds connection tag between the concepts which have relations $\mathcal{F}_{m}$ or $\mathcal{L}_{m}$ respectively. We use $\mathcal{F}_{c d}$ to denote fusion connection of direct mapping, $\mathcal{F}_{c s}$ to denote fusion connection of subsumption mapping and $\mathcal{F}_{c c}$ to denote the fusion connection of composition or decomposition mapping. The fusion connection list is a list and its elements are the fusion connections, denote it as $F L$.

1. The first step of ontology integration is Ontology Fusion for Direct Mapping, which creates fusion list $F L$ from the mapping list of different local ontologies, it can be described by algorithm 1 .

2. The second step of ontology integration is Ontology Fusion for Complex Semantic Mapping, which used to find the mappings of the concept that are not in the mapping list, the basic idea of this step is to find the semantic similarity of the mapping relations, and create new mappings between these relations. We propose this step in two situations, one is to find the mapping between two subsumption mapping relations, and the other is to find the mapping between two composition mapping relations or decomposition mapping relations.

We propose subsumption mapping situation at first, suppose $\mathcal{M}_{1}$ and $\mathcal{M}_{2}$ are two subsumption mappings, and $R_{1}$ is the mapping relation of $\mathcal{M}_{1}, C_{10}$ is the concept of source ontology, $C_{1 i}(1 \leq i \leq n)$ is the concept of target ontology; $R_{2}$ is the mapping relation of $\mathcal{M}_{2}, C_{20}$ is the concept of source ontology, $C_{2 j}$ $(1 \leq j \leq m)$ is the concept of target ontology . If there exists $J S\left(R_{1}, R_{2}\right) \geq 1-\epsilon$, it means that these two relations of the mappings can match each other, we create a new mapping named $M^{\prime}$ and create the fusion connection list $F L$ with algorithm 2 .

Then we propose the composition and decomposition mapping situation, these kinds of mapping are very complex because the concatenations between two concepts of target ontology are different with each other, we divide the concatenation of target concepts into two kinds. One kind of concatenation does not have meanings, just means that the concatenation between these concepts exists(maybe most of the concatenation can be expressed as this kind). For example the mapping: address=contact (country, state, city, street, postcode), the concatenation 


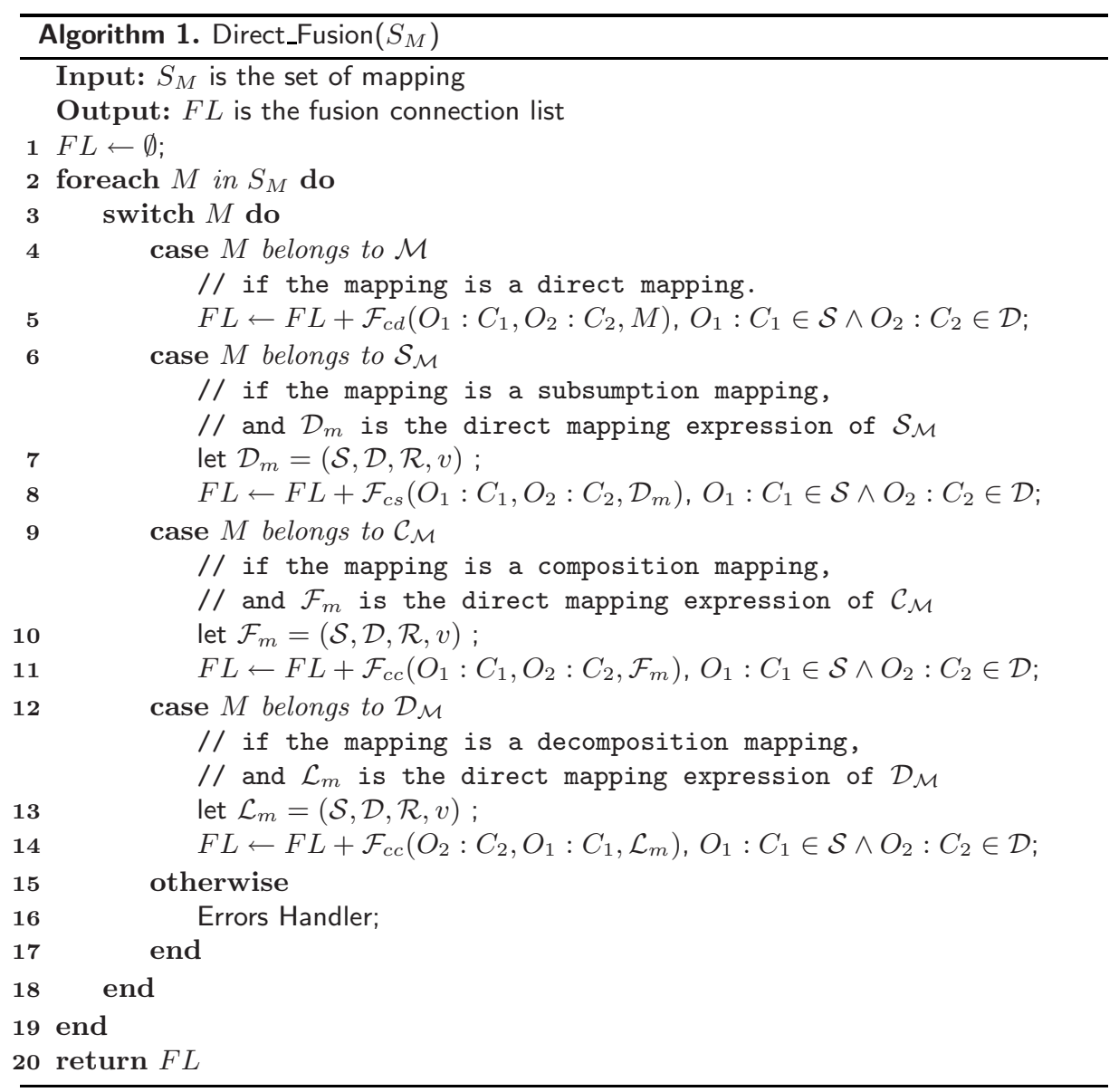

among contact, country, state, city, street and postcode are this kinds of concatenations. We name this kind of concatenation as None-Meanings Concatenation; The other kind of concatenation does have meanings between the concepts, we name it as Full-Meanings Concatenation. For example, the mapping payment $=$ total $($ commodity_amount $*($ unit_price + tax $))$ is this kind of concatenation. For the first kind of concatenation, we only need to identify whether the related concepts satisfy the definition of semantic similarity or not, if they satisfy the definition, add a new fusion connection to the fusion connection list. Before we discuss the other kind of concatenation, we introduce the concept of Fusion Equivalence and Fusion Subsumption.

For composition (or decomposition) mappings $\mathcal{M}_{1}$ and $\mathcal{M}_{2}, \mathcal{A}_{m 1}$ and $\mathcal{A}_{m 2}$ represents the concatenation relationship of $\mathcal{M}_{1}$ and $\mathcal{M}_{2}$ respectively, we use $\left|\mathcal{A}_{m 1}\right|$ and $\left|\mathcal{A}_{m 2}\right|$ to denote the amount of the concepts in $\mathcal{A}_{m 1}$ and $\mathcal{A}_{m 2}, \mathcal{A}_{m 1}$ and $\mathcal{A}_{m 2}$ are Fusion Equivalence iff, 


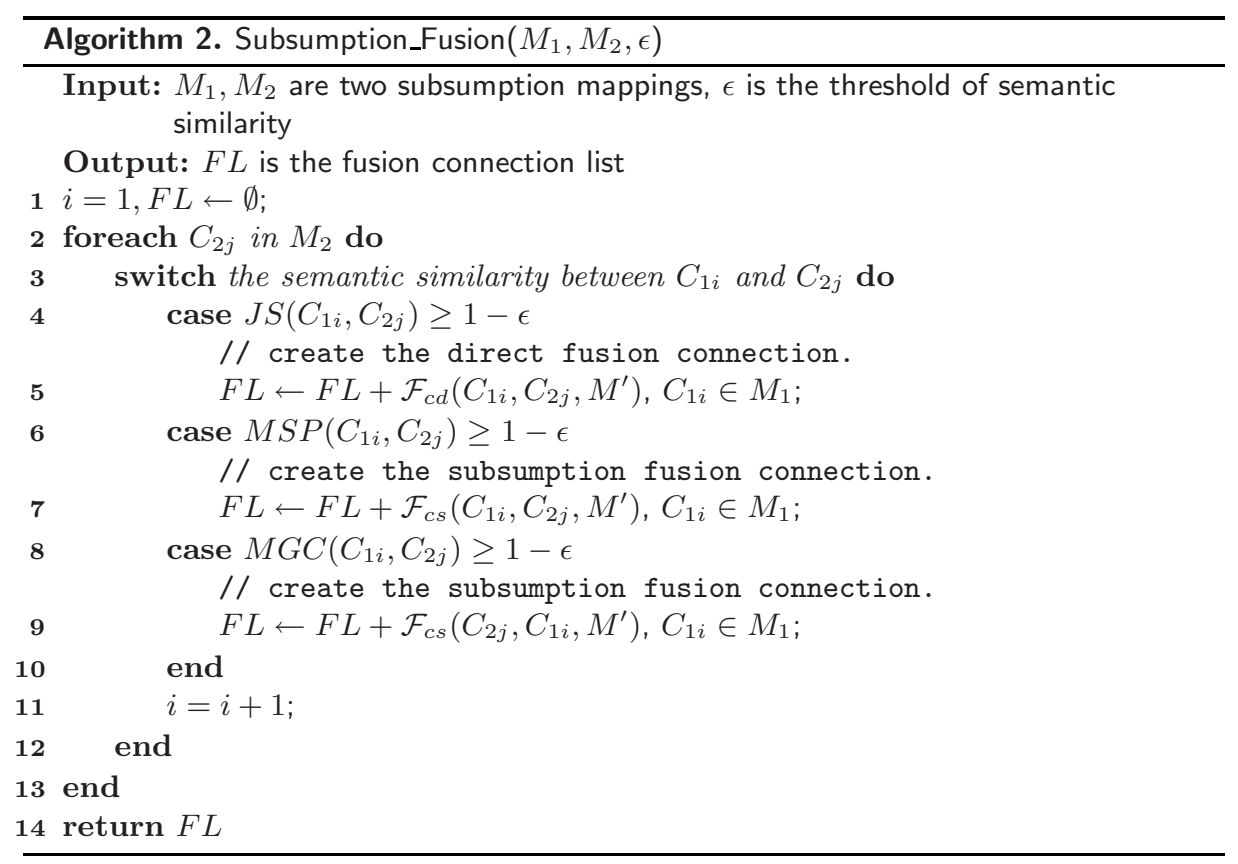

i). $\mathcal{A}_{m 1}$ and $\mathcal{A}_{m 2}$ are Full-Meaning Concatenations, and $\left|\mathcal{A}_{m 1}\right|=\left|\mathcal{A}_{m 2}\right|$;

ii). For $\forall i\left(C_{1 i} \sqsubseteq \forall\right.$ Concatenation $_{1, i+1} \cdot C_{1, i+1}, C_{2 i} \sqsubseteq \forall$ Concatenation $_{2, i+1}$. $\left.C_{2, i+1}\right)$ exists $\left(J S\left(C_{1 i}, C_{2 i}\right) \geq 1-\epsilon\right) \wedge\left(J S\left(C_{1, i+1}, C_{2, i+1}\right) \geq 1-\epsilon\right) \wedge$ $\left(J_{S}\left(\right.\right.$ Concatenation $_{1, i+1}$, Concatenation $\left.\left._{2, i+1}\right) \geq 1-\epsilon\right)$.

We use $\mathcal{A}_{m 1} \Leftrightarrow \mathcal{A}_{m 2}$ to denote the fusion equivalence of $\mathcal{A}_{m 1}$ and $\mathcal{A}_{m 2}$, if $\left|\mathcal{A}_{m 1}\right|>\left|\mathcal{A}_{m 2}\right|$, suppose $\left|\mathcal{A}_{m 1}\right|=m$ and $\left|\mathcal{A}_{m 2}\right|=n$, then $m>n$. If $\mathcal{A}_{m 1, n}$ is the first $n$ concepts of $\mathcal{A}_{m 1}$ and $\mathcal{A}_{m 1, n} \Leftrightarrow \mathcal{A}_{m 2}$, then we say $\mathcal{A}_{m 1}$ and $\mathcal{A}_{m 2}$ are Fusion Subsumption, denotes it as $\mathcal{A}_{m 1} \Rightarrow \mathcal{A}_{m 2}$, or $\mathcal{A}_{m 2} \Rightarrow \mathcal{A}_{m 1}$ if $n>m$.

We use $A U_{1}$ and $A U_{2}$ to denote the concepts of $\mathcal{A}_{m 1}$ and $\mathcal{A}_{m 2}$ respectively, and whose concatenations are None-Meanings Concatenations, use $A N_{1}$ and $A N_{2}$ to denote the concepts whose concatenations are Full-Meanings concatenations. We use $A E_{1}$ and $A E_{2}$ to denote the concepts of $A N_{1}$ and $A N_{2}$, which keep fusion equivalence or fusion subsumption relationship with each other. We can express the fusion connection with algorithm 3 .

3. The last step of ontology integration is Canonical Fusion, which merges the concepts of the fusion connection into one concept if the fusion connection type is $\mathcal{F}_{c d}$ or $\mathcal{F}_{c c}$, and adds a real relation connection to the concepts if the fusion connection type is $\mathcal{F}_{c s}$. For example the fusion connection $\mathcal{F}_{c d}=\left(C_{1}, C_{2}, M\right)$, $C_{1}$ and $C_{2}$ are concepts of different ontologies, they will be merged to a concept $\left(C_{1}, C_{2}\right)$ and all the hierarchy of the concepts will be kept. But not all the concepts with the same mapping relation can be merge into one concepts, only the concepts which have strong mapping relation can be merged. For example, if the 


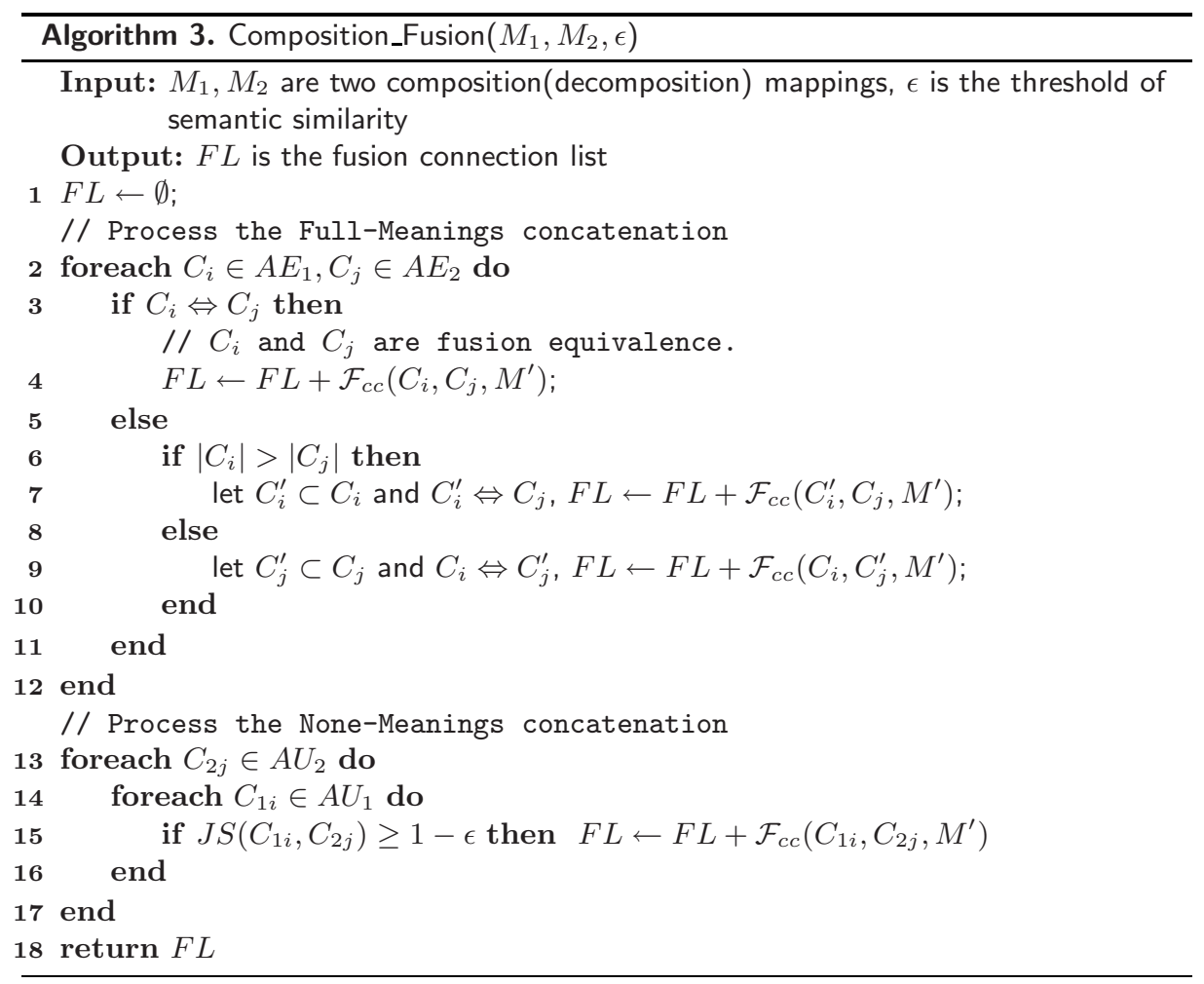

mappings $\mathcal{M}\left(C_{1}, C_{2}, \mathcal{R}, v_{1}\right), \mathcal{M}\left(C_{2}, C_{3}, \mathcal{R}, v_{2}\right)$ and $\mathcal{M}\left(C_{1}, C_{3}, \mathcal{R}, v_{3}\right)$ satisfy the strong mapping property, we can merge the concept $C_{1}, C_{2}, C_{3}$ into one concept $\left(C_{1}, C_{2}, C_{3}\right)$, otherwise, we have to merge them into two concepts $\left(C_{1}, C_{2}\right)$ and $\left(C_{2}, C_{3}\right)$.

\section{Discussion and Conclusion}

Semantic data grid service mechanism we present in this paper wrapped various information source through semantic fusion mechanism, and used MediatorWrapper to support the heterogeneous data source, employed mediator structure to realize virtual data gird service which supports semi-structured information retrieving language.

Because XML is rapidly becoming a language of choice to express, store and query information on the web, other kinds of web information such as HTML-based web information can be transferred to XML based information with annotation technologies. Users can query information with XML languages, XPath based XML query style language to retrieve information from different grid nodes, languages such as XQuery, XUpdate are suitable for retrieving 
information in distributed integration systems. It is suitable to extend XML query algebra to support semantic querying on the gird, some works have focused on this topic [9].

\section{Acknowledgment}

This work was partially supported by NSF (Natural Science Fundation) of China under grant number 60425206, and it was partially supported by China Postdoctoral Science Foundation under grant number 20060400275 and Jiangsu Postdoctoral Science Foundation under grant number 0601009B. It was partially supported by a grant from the NSF of Hubei Prov. of China under grant number $2005 \mathrm{ABA} 235$.

\section{References}

1. Levy, A.Y., Rajaraman, A., Ordille, J.J.: Query heterogeneous information sources using source descriptions. In: Proceedings of the 22nd VLDB Conference, Mumbai, India, Morgan Kaufmann Publishers Inc (1996) 251-262

2. Antonioletti, M., Atkinson, M., Baxter, R., et al.: The design and implementation of Grid database services in OGSA-DAI. Concurrency and Computation: Practice and Experience 17 (2005) 357-376

3. Wöhrera, A., Brezanya, P., Tjoab, A.M.: Novel mediator architectures for Grid information systems. Future Generation Computer Systems 21 (2005) 107-114

4. Zhuge, H., Liu, J.: A Knowledge Grid Operation Language. ACM SIGPLAN Notices 38 (2003) $57-66$

5. Gu, J., Xu, B.: XML based Semantic Query Mechanism on Grid. In: ISPA 2006 Workshops, LNCS 4331, Springer Verlag (2006) 532-541

6. Sheng, Q.J., Shi, Z.Z.: A Knowledge-based Data Model and Query Algebra for the Next-Gereration Web. In: Proceedings of APWeb 2004, LNCS 3007 (2004) 489-499

7. Gu, J., Zhou, Y.: Ontology Fusion with Complex Mapping Patterns. In: Proceedings of 10th International Conference on Knowledge-Based, Intelligent Information and Engineering Systems, Bournemouth, United Kingdom, LNCS 4251, Springer Verlag (2006) 738-745

8. KWON, J., JEONG, D., LEE, L.S., BAIK, D.K.: Intelligent semantic concept mapping for semantic query rewriting/optimization in ontology-based information integration system. International Journal of Software Engineering and Knowledge Engineering 14 (2004) 519-542

9. Gu, J., Hu, B., Zhou, Y.: Semantic Query Planning Mechanism on XML based Web Information Systems. In: WISE 2006 Workshops, Wuhan, China, LNCS 4256, Springer Verlag (2006) 194-205 